\title{
Estimation de la sédimentation dans la retenue d'eau de la rivière Lobo à l'aide de la théorie des bassins de décantation
}

\author{
Bérenger KOFFI ${ }^{1,2}$, Kouakou Lazare KOUASSI ${ }^{1}$, Martin SANCHEZ ${ }^{2}$, \\ Zilé Alex KOUADIO ${ }^{1}$, Kouakou Hervé KOUASSI ${ }^{1}$, Affoué Berthe YAO ${ }^{1}$
}

\section{Laboratoire des Sciences et Technologies de l'Environment, Université Jean} Lorougnon Guédé, BP 150 Daloa, Côte d'Ivoire.

2. Laboratoire de Planétologie et Geodynamique (LPG), Université de Nantes, Faculté des Sciences et Techniques, Nantes, France.

Koffiberen@gmail.com

\section{Résumé :}

Ce travail aborde le problème d'accessibilité à l'eau potable de la troisième plus grande ville de Côte d'Ivoire. Sur le bassin versant de la rivière Lobo, le problème se pose avec plus d'acuité puisque la retenue aménagée pour l'alimentation en eau des populations de la ville de Daloa fait face au phénomène d'eutrophisation et de sédimentation. Ces problèmes entraînent une réduction de la capacité de stockage de la retenue d'eau. Une étude préliminaire de la dynamique des sédiments cohésifs est étudiée. Le comblement de cette retenue est évalué à l'aide de six modèles hydro-sédimentaires issus de la théorie des bassins de décantation en tenant compte de la vitesse de chute des particules en suspension et des caractéristiques du bassin versant. La concentration moyenne de la rivière Lobo est estimée à $0,0138 \mathrm{~kg} / \mathrm{m}^{3}$ avec des apports solides compris entre 87,4 et $1074,2 \mathrm{t} /$ mois transitant dans la retenue. Selon les résultats des modèles, la retenue d'eau de la rivière Lobo subit un envasement dont l'épaisseur des dépôts fluctue entre 0,58 et $12,43 \mathrm{~mm} / \mathrm{mois}$. L'efficacité de la retenue est comprise entre 92,5 et $100 \%$ pour une vitesse de chute constante et de 39 à $59 \%$ dans le cas d'une vitesse de chute variable des matières en suspension.

Mots-clés : Matières en suspension, Sédiments cohésifs, Vitesse de chute, Décantation, Erosion, Envasement, Rivière Lobo.

\section{Introduction}

Le vieillissement naturel des plans d'eau par eutrophisation qui devrait se dérouler sur quelques centaines voire des milliers d'années a lieu de manière précoce et souvent dramatique dans les petites retenues d'eau en Afrique de l'Ouest (MAIGA et al., 2001). Les processus de sédimentation sont de plus en plus accentués par les pressions anthropiques (MOURIS et al., 2018). Ainsi, les zones aménagées pour le captage des eaux de surface sont de plus en plus menacées par les phénomènes d'envasement. La retenue d'eau de la rivière Lobo, source d'approvisionnement en eau potable de la commune de Daloa dans le Centre-Ouest de la Côte d'Ivoire, ne fait pas exception à la règle. Le plan 


\section{Thème 2 - Dynamique sédimentaire}

d'eau est envahi par des macrophytes avec comme conséquences, la dégradation de la qualité de l'eau et le comblement par la biomasse issue de ces macrophytes (GROGA et al., 2017). A la prise d'eau de la rivière Lobo, l'évolution progressive des sédiments réduit la capacité de stockage de la retenue et a amené les gestionnaires de production d'eau potable de la ville à effectuer un dragage en 2011, afin de redonner une profondeur suffisante au réservoir. Malgré les efforts consentis par les autorités compétentes, la retenue d'eau de la rivière Lobo est toujours confrontée à des problèmes d'envasement. L'objectif de cette étude est d'établir un modèle hydro-sédimentaire à l'aide de la théorie des bassins de décantation afin de quantifier les dépôts de sédiments dans la retenue d'eau de la rivière Lobo pour une meilleure gestion du système d'alimentation en eau potable de la ville de Daloa.

\section{Site d'étude}

La retenue d'eau de la rivière Lobo est située à $25 \mathrm{~km}$ de la ville de Daloa. Le bassin versant de la rivière Lobo a une superficie de $7280 \mathrm{~km}^{2}$ à la station hydrométrique de Nibéhibé. Il est situé entre les $6^{\circ} 2$ et $7^{\circ} 55$ de longitude Ouest et $6^{\circ}$ et $6^{\circ} 55$ de latitude Nord, voir figure 1.

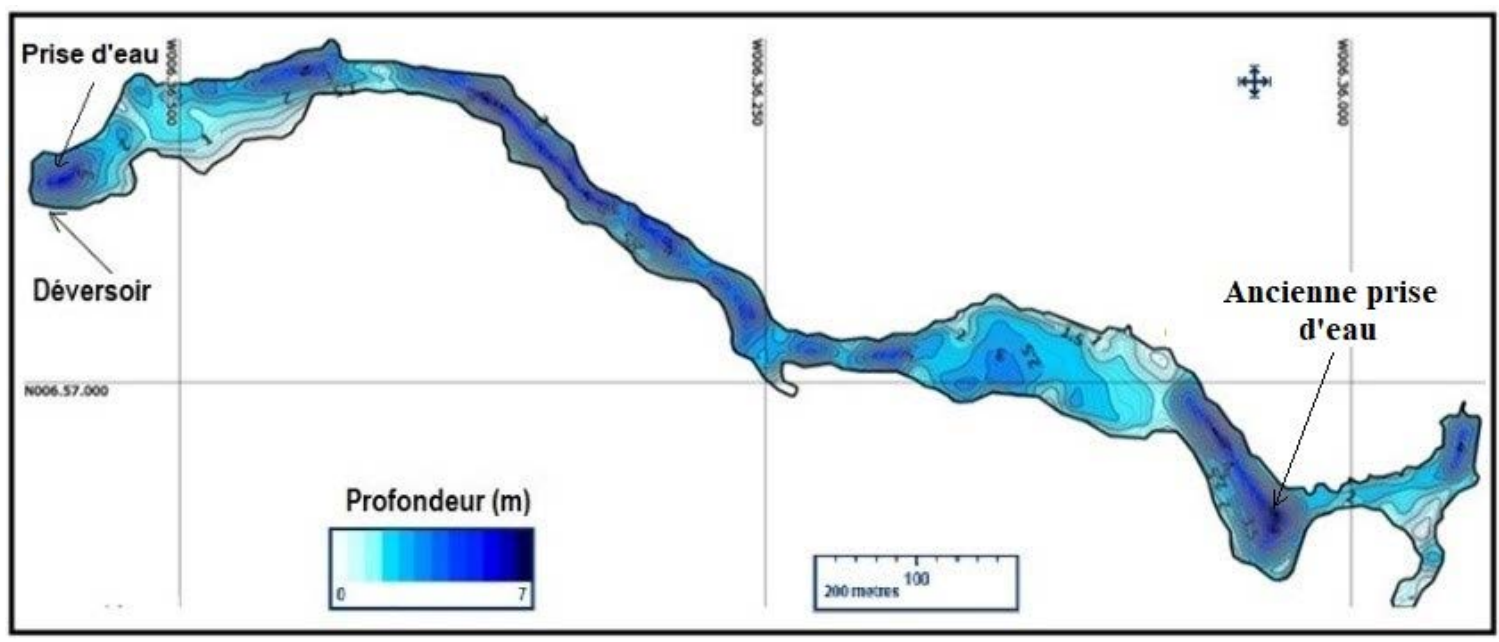

Figure 1. Présentation de la retenue d'eau de la rivière Lobo.

La retenue est la principale source d'approvisionnement en eau potable de la ville. Elle a été construite depuis 1976 (MAIGA et al., 2001). Le débit moyen de la rivière à la station hydrométrique de Nibéhibé est de $12,43 \mathrm{~m}^{3} / \mathrm{s}$.

Avec une pluie moyenne annuelle de 1254,6 mm (figure 2), la saison pluvieuse débute de Mars à Octobre et une saison sèche va de Novembre à Février, sur le bassin. 


\section{XVI'̀mes Journées Nationales Génie Côtier - Génie Civil \\ Le Havre 2020}

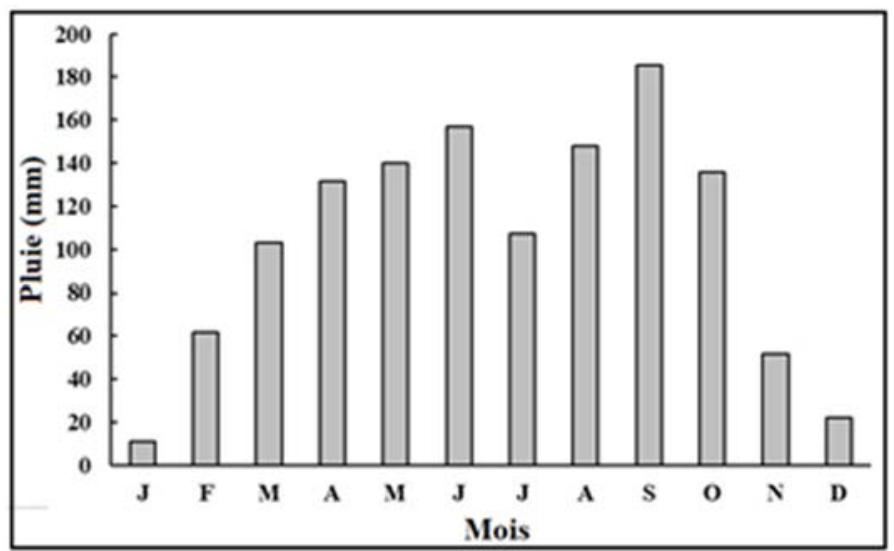

Figure 2. Pluie moyenne à la station synoptique de Daloa sur la période (1966-2017).

\section{Méthodes}

\subsection{Evaluation du transport solide en suspension}

L'évaluation du transport des sédiments en suspension dans la retenue d'eau de la rivière Lobo a été effectuée par le prélèvement d'échantillons d'eau dans la retenue et à la station de Sikaboutou en amont de la retenue. En moyenne, 20 échantillons d'eau ont été prélevés à différentes profondeurs (en surface, à mi profondeur et au fond) à l'aide d'une bouteille hydrologique de marque Van Dorn du mois d'Avril à Décembre 2019. Pour des raisons de disponibilité de données de débit sur tous les mois, nous évaluerons le transport solide sur trois mois (Octobre-Novembre-Décembre). Au laboratoire, les échantillons d'eau ont été filtrés sur une rampe de filtration millipore à l'aide de filtres WHATMAN GF/F circulaire de $47 \mathrm{~mm}$ de diamètre et de porosité $0,45 \mu \mathrm{m}$ préalablement pesés. Après séchage à $105^{\circ} \mathrm{C}$, les filtres ont été soigneusement récupérés et repesés afin de déterminer les concentrations totales de matières en suspension exprimées en $\mathrm{kg} / \mathrm{m}^{3}$. Le débit solide $\left(Q_{s}(\mathrm{~kg} / \mathrm{s})\right)$ est obtenu en multipliant le débit liquide $Q_{L}\left(\mathrm{~m}^{3} / \mathrm{s}\right)$ par la concentration de matières en suspension $C\left(\mathrm{~kg} / \mathrm{m}^{3}\right)$ :

$Q_{s}=C \times Q_{L}$

Le taux d'érosion spécifique est obtenu par le rapport des apports solides $(A S)$ sur la superficie du bassin versant de la rivière Lobo.

\subsection{Mesure de la répartition de la vitesse de chute in situ en tube de sédimentation}

Dans le but d'étudier la répartition verticale des matières en suspension (MES) et leurs vitesses de chute en condition d'eau calme, trois tests ont été effectués sur les échantillons d'eau prélevés durant les mois d'Octobre, Novembre et Décembre. La distribution locale des MES en termes de vitesses de chute a été examinée par la méthode du tube d'Owen. Les concentrations en MES des échantillons sont mesurées par la méthode de filtration au laboratoire. Grâce aux mesures au tube d'Owen, une courbe de repartition de la vitesse de chute des MES est obtenue. 


\section{Thème 2 - Dynamique sédimentaire}

\subsection{Modélisation du taux de sédimentation dans la retenue}

Une modélisation de la sédimentation des MES dans la zone de décantation de la retenue d'eau de la la rivière Lobo est réalisée. Deux grands types de modélisations ont été devéloppés à savoir :

- Vitesse de chute constante ;

- Vitesse de chute distribuée selon la loi gamma.

Nous sommes partis d'un cas classique en supposant que la retenue d'eau est de forme rectangulaire, avec des profils en travers identitiques et une profondeur moyenne mensuelle de la retenue. Comme données de terrain, 26 profils en travers de la retenue ont été réalisés sur le long de la retenue. Les paramètres utilisés sont listés dans le tableau 1 suivant.

Tableau 1. Caractéristiques de la retenue et du bassin versant de la rivière Lobo.

\begin{tabular}{lll}
\hline Paramètres & Valeurs & Unités \\
\hline Superficie du bassin versant à Nibéhibé & 7280 & $\mathrm{~km}^{2}$ \\
Superficie du bassin versant à la retenue & 5740 & $\mathrm{~km}^{2}$ \\
Longueur moyenne de la retenue $(L)$ & 1331 & $\mathrm{~m}$ \\
Largeur moyenne de la retenue $(B)$ & 43 & $\mathrm{~m}$ \\
Concentration moyenne de la rivière & 0,0138 & $\mathrm{~kg} / \mathrm{m}^{3}$ \\
Masse volumique sèche de dépôt $\left(\rho_{\mathrm{s}}\right)$ & 1421 & $\mathrm{~kg} / \mathrm{m}^{3}$ \\
\hline
\end{tabular}

3.3.1 Vitesse de chute constante sans turbulence (Modèle VC-ST)

La concentration près du fond reste constante jusqu'à la décantation totale des matières en suspension si on considère une vitesse de chute constante et un écoulement sans mélange dans la retenue d'eau de la rivière Lobo. Cependant, la concentration en surface est nulle et l'interface entre la concentration nulle et la concentration au fond se propage de la surface vers le fond avec la même vitesse chute que les matières en suspension (SANCHEZ, 2018).

\subsubsection{Vitesse de chute constante et mélange avec modèle de Teeter (Modèle VC-MT)}

Une grande partie de l'action dans la dynamique des sédiments fins se produit à l'interface suspension/lit de sédiments. TEETER (1986) a établi une relation entre la concentration à proximité du lit et le nombre de Peclet $(\mathrm{Pe})$ par des approches analytiques et numériques pour examiner les relations de transport vertical. La variation verticale des matières en suspension dans la retenue d'eau de la rivière Lobo pour une vitesse de chute constante en présence de turbulence est analysée dans cette note. 


\section{XVI'èmes Journées Nationales Génie Côtier - Génie Civil \\ Le Havre 2020}

\subsubsection{Vitesse de chute constante et mélange complet (Modèle VC-MC)}

Un mélange parfait des matières en suspension avec une concentration constante sur toute la profondeur d'eau et une vitesse de chute constante dans la retenue est considéré. Cela nous permet d'obtenir une solution analytique de l'évolution de la concentration des matières en suspension sur chaque profil en travers et l'éfficacité de la retenue (SANCHEZ, 2018).

\subsubsection{Vitesse de chute distribuée (loi Gamma) sans turbulence (Modèle VD-ST)}

En condition d'eau calme, plusieurs lois de distribution de la vitesse de chute peuvent être utilisées pour modéliser la repartition des vitesses de chute des MES, mais la loi gamma est celle qui se rapproche le plus de la réalité (SANCHEZ, 2006). Après un laps de temps, on suppose qu'une fraction des particules se dépose dans la retenue. En absence de turbulence, avec une concentration constante sur toute la profondeur, la variation de la concentration moyenne des matières en suspension selon l'horizontale a été démontrée par SANCHEZ (2018).

\subsubsection{Vitesse de chute distribuée (loi Gamma) et mélange avec modèle de Teeter (Modèle} VD-MT)

Les hypothèse imposées dans cette note sont celles de TEETER (1986) et de la loi de distribution gamma (SANCHEZ, 2006). En effet, selon la loi gamma, toutes les particules se déposent avec une vitesse de chute variable en suivant une loi stochastique. Une solution analytique de la concentration moyenne des MES dans la retenue est obtenue. La capacité de la retenue à piéger les apports solides qui transitent dans le réservoir de la zone de captage de la rivière Lobo est également analysée.

\subsubsection{Vitesse de chute distribuée (loi Gamma) et mélange complet (Modèle VD-MC)}

Le processus de sédimentation en fonction de la vitesse de chute locale de la masse de sédiments en suspension suit une loi de distribution gamma (SANCHEZ, 2006). Le paramètre $r$ et la vitesse moyenne de chute suffisent pour définir une distribution gamma. En effet, en présence de turbulence, avec une concentration constante sur toute la profondeur, la variation de la concentration moyenne des matières en suspension et l'éfficacité des bassins de décantation ont été démontrées par SANCHEZ (2018).

\section{Résultats}

\subsection{Evaluation du transport solide en suspension}

Les résultats montrent que les eaux sont plus chargées en matériaux solides principalement durant la période des crues (Octobre). On observe une baisse de la concentration de matières en suspension entre Octobre et Décembre avec respectivement des concentrations de 0,$0180 ; 0,0130$ et $0,0102 \mathrm{~kg} / \mathrm{m}^{3}$. Les apports solides sont compris 


\section{Thème 2 - Dynamique sédimentaire}

entre 87,4 et 1074,2 t/mois avec des apports plus importants en Octobre comparativement au mois de Décembre (tableau 2). Cependant, les plus faibles apports solides ont été observés durant le mois de Décembre qui marque le début de la saison sèche. On constate que le taux d'érosion spécifique du bassin évolue dans le même sens que les apports solides avec des valeurs comprises entre 0,0120 et $0,1476 \mathrm{t} / \mathrm{km}^{2} / \mathrm{mois}$.

Tableau 2. Apports solides et taux d'érosion spécifique sur le bassin versant de la rivière Lobo.

\begin{tabular}{llll}
\hline Paramètres & Octobre & Novembre & Décembre \\
\hline MES $\left(\mathbf{k g} / \mathbf{m}^{3}\right)$ & 0,0180 & 0,0130 & 0,0102 \\
Débit liquide $\boldsymbol{Q}_{\mathbf{L}}\left(\boldsymbol{m}^{3} / \mathbf{s}\right)$ & 22 & 8,0 & 3,2 \\
$\boldsymbol{Q}_{\boldsymbol{s}}(\mathbf{t} / \mathbf{m o i s})$ & 1074,2 & 280,7 & 87,4 \\
Superficie bassin versant $\left(\mathbf{k m}^{2}\right)$ & 5740 & 5740 & 5740 \\
$\boldsymbol{T}\left(\boldsymbol{t} / \mathbf{k m}^{2} / \mathbf{m o i s}\right)$ & 0,1871 & 0,0489 & 0,0152 \\
\hline
\end{tabular}

4.2 Résultats de la répartition de la vitesse de chute et des données d'entrée des modèles La figure 3 montre les résultats des fonctions de répartition mensuelle (Octobre, Novembre, Décembre) de la vitesse de chute des MES. Avec une concentration moyenne de $0,0138 \mathrm{~kg} / \mathrm{m}^{3}$, on obtient une vitesse de chute moyenne de $0,994 \mathrm{~mm} / \mathrm{s}$ pour le mois d'Octobre, $0,562 \mathrm{~mm} / \mathrm{s}$ pour le mois de Novembre et $0,496 \mathrm{~mm} / \mathrm{s}$ pour le mois Décembre. Les vitesses de chute médianes $\left(\mathrm{W}_{50 \%}\right)$ sont comprises entre 0,058 et $0,08 \mathrm{~mm} / \mathrm{s}$ avec un paramètre $r$ compris entre 0,182 et 0,214 (tableau 3 ). La valeur retenue du coefficient de Strickler local pour un dépôt sable fin-vase est $K_{s}=43 \mathrm{~m}^{1 / 3} \mathrm{~s}^{-1}$.

Tableau 3. Résultats des paramètres d'entrée des modèles, (Note : $k=0,067$ ).

\begin{tabular}{|c|c|c|c|}
\hline Paramètres & Octobre & Novembre & Décembre \\
\hline$V_{50}(\mathrm{~mm} / \mathrm{s})$ & 0,08 & 0,07 & 0,058 \\
\hline$V_{90}(\mathrm{~mm} / \mathrm{s})$ & 3,0 & 1,7 & 1,5 \\
\hline$V_{90} / V_{50}$ & 37,5 & 24,3 & 25,9 \\
\hline$r$ & 0,1827 & 0,2148 & 0,2093 \\
\hline$V_{\text {moy }} / V_{50}$ & 12,43 & 8,03 & 8,57 \\
\hline$V_{\text {moy }}(m m / s)$ & 0,9944 & 0,5621 & 0,49706 \\
\hline Débit liquide : $Q_{L},\left(m^{3} / s\right)$ & 22 & 8,0 & 3,2 \\
\hline Profondeur moyenne : $d,(m)$ & 3,2 & 2,58 & 2,41 \\
\hline Vitesse d'écoulement : $u=Q_{L} /(B d),(m / s)$ & 0,160 & 0,072 & 0,031 \\
\hline Vitesse critique : $v_{c}=Q_{L} /(B L),(\mathrm{mm} / \mathrm{s})$ & 0,3844 & 0,14 & 0,056 \\
\hline$V_{m o y} / v_{c}$ & 2,59 & 4,02 & 8,87 \\
\hline Coefficient de frottement local : $C_{f}=2 g /\left(K_{s}^{2} d^{1 / 3}\right)$ & 0,0072 & 0,0078 & 0,0079 \\
\hline Vitesse de cisaillement : $u^{*}=\left(C_{f} / 2\right)^{1 / 2} u,(\mathrm{~mm} / \mathrm{s})$ & 9,59 & 4,48 & 1,94 \\
\hline Nombre de Peclet : Pe $=V_{m o y} /(\mathbf{k u} *)$ & 1,55 & 1,87 & 3,83 \\
\hline
\end{tabular}




\section{XVİ̀mes Journées Nationales Génie Côtier - Génie Civil \\ Le Havre 2020}

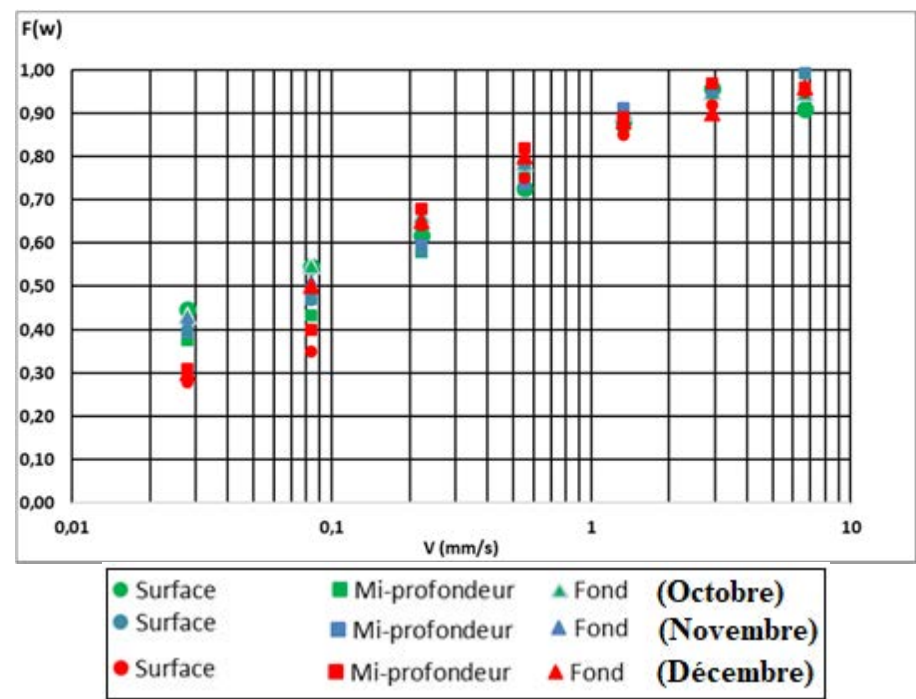

Figure 3. Fonctions de répartition de la vitesse de chute des matières en suspension pour les mois d'Octobre, Novembre et Décembre.

4.3 Masse et épaisseur des dépôts dans la retenue d'eau de la rivière Lobo

Les différents résultats des modèles montrent que les dépôts varient en fonction des mois. Pendant la période des hautes eaux (Octobre), il semble que les dépôts soient plus importants que pendant la période des basses eaux (Novembre-Décembre) (tableau 4). Les taux de dépôt les plus élevés sont observés durant le mois d'Octobre avec une épaisseur de dépôts comprise entre 1,96 et $12,7 \mathrm{~mm} / \mathrm{mois}$ et les plus faibles taux sont observés durant les mois de Décembre avec des épaisseurs de dépôts comprises entre 0,31 et $1,06 \mathrm{~mm} / \mathrm{mois}$. Les résultats des modèles VC-MT et VC-MC sont pratiquement dans le même ordre de grandeur.

Tableau 4. Masse et épaisseur des dépôts obtenues dans la retenue de la rivière Lobo.

\begin{tabular}{clccc}
\hline & Modèles & Octobre & Novembre & Décembre \\
\hline & Modèle VC-ST & 18,67 & 4,80 & 1,56 \\
Masse de & Modèle VC-MT & 17,52 & 4,74 & 1,56 \\
dépôts & Modèle VC-MC & 16,11 & 4,63 & 1,56 \\
(kg/m²/mois) & Modèle VD-ST & 5,17 & 1,67 & 0,68 \\
& Modèle VD-MT & 3,21 & 1,10 & 0,54 \\
& Modèle VD-MC & 2,88 & 0,97 & 0,45 \\
\hline \multirow{2}{*}{ Epaisseur des } & Modèle VC-ST & 13,1 & 3,38 & 1,10 \\
dépôts & Modèle VC-MT & 12,33 & 3,34 & 1,10 \\
& Modèle VC-MC & 11,34 & 3,26 & 1,10 \\
& Modèle VD-ST & 3,64 & 1,67 & 0,68 \\
& Modèle VD-MT & 2,88 & 0,97 & 0,45 \\
& Modèle VD-MC & 2,03 & 0,69 & 0,32 \\
\hline
\end{tabular}




\section{Thème 2 - Dynamique sédimentaire}

\subsection{Efficacité de la retenue}

La capacité de la retenue à piéger les sédiments dépend de la vitesse de chute, de la vitesse critique et du paramètre $r$. On observe une bonne synchronisation entre nos modèles et celui proposé par TEETER (1986). Cette bonne synchronisation avec le modèle proposé de TEETER (1986), montre une fiabilité de notre modèle. Cette continuité parfaite entre les points tracés correspond à un dépôt avec un tassement constant. On constate que pour une vitesse de chute constante avec $\bar{V} / v_{c} \geq 4$, presque tous les sédiments (92,5-100\%) ont tendance à être piégés par la retenue d'eau.
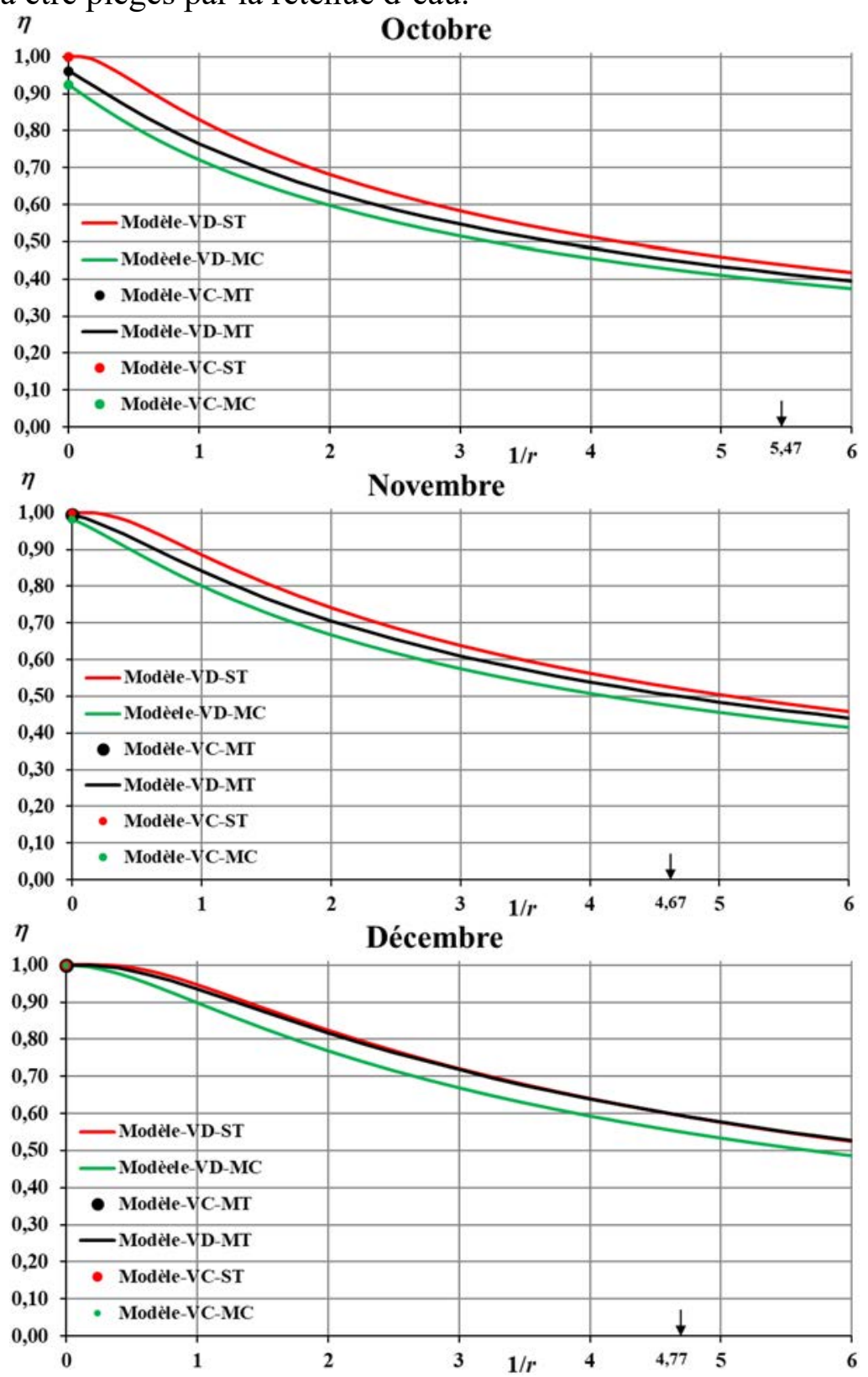

Figure 4. Evolution de l'efficacité ( $\eta$ ) de la retenue d'eau de la rivière Lobo pour les mois d'Octobre, Novembre et Décembre en fonction de $1 / r=(\sigma / \bar{V})^{2}$. 


\section{XVI'̀mes Journées Nationales Génie Côtier - Génie Civil \\ Le Havre 2020}

Cependant, pour une vitesse de chute variable, la capacité de la retenue à piéger les sédiments est comprise entre 39 et 59\%. La capacité de la retenue à piéger le maximum de sédiments est, observée pendant le mois de décembre avec des valeurs comprises entre 59 et $100 \%$ (figure 4). Cependant, le plus faible taux d'efficacité est observé durant les mois d'Octobre avec des valeurs comprises entre 39 et 100\%. Il faut noter que le modèle VC-MT et le modèle VC-MC donnent sensiblement les mêmes résultats d'efficacité.

\section{Discussion}

Sur la base des campagnes de terrain réalisées sur la rivière Lobo, les dépôts mensuels dans la retenue d'eau ont été estimés à l'aide de six modèles en fonction de la répartition de la vitesse de chute des sédiments. Les résultats montrent que les apports solides sont plus importants pendant le mois d'Octobre (hautes eaux) que pendant la saison sèche (Novembre-Décembre). Cette situation peut s'expliquer par le fait que pendant les saisons des pluies, les flux transportent beaucoup de sédiments en raison des apports plus importants. BOUGUERRAS \& BOUANANI (2019) rapportent que l'importance et la nature des débits solides en suspension sont liées d'une part à l'intensité de l'érosion par l'eau de ruissellement, donc à l'importance des précipitations et, d'autre part, à la capacité de l'écoulement à transporter les matières solides. L'analyse de la vitesse de chute par une fonction de répartition des matières en suspension montre que la vitesse de chute médiane est nettement inférieure à la vitesse de chute moyenne. Selon SANCHEZ (2006), cette augmentation pourrait être due au phénomène de turbulence qui augmente de façon très nette avec la vitesse de chute des matières des matières en suspension. Les dépôts mensuels ont été calculés en fonction des mesures de la vitesse de chute et des débits mensuels obtenus sur trois mois, à savoir durant la période des hautes eaux (Octobre), et pendant la saison sèche (Novembre-Décembre). La quantité des dépôts varie globalement entre 0,44 et $18,06 \mathrm{~kg} / \mathrm{m}^{2} /$ mois ou en termes d'épaisseur entre 0,31 et $12,7 \mathrm{~mm} / \mathrm{mois}$. Les valeurs de la sédimentation obtenues dans cette modélisation sont un peu plus faibles que les valeurs de KOUASSI et al. (2013b). Cette différence peut s'expliquer par le fait que, dans le cas de cette étude, la vitesse de chute est considérée comme une variable stochastique. Aussi, son étude a été réalisée avec une plage de données sur toutes les saisons, ce qui n'est pas le cas pour cette étude. Les apports fluviatiles plus importants observés sur le fleuve Bandama pourraient être l'une des causes de cette différence.

\section{Conclusion}

Dans le cadre de cette étude, nous nous sommes intéressés au processus final de décantation et de dépôt des sédiments qui ont été piégés et maintenus en suspension dans la retenue d'eau de la rivière Lobo. À cet effet, en se basant sur trois paramètres essentiels à savoir la vitesse de chute, la concentration des matières en suspension et le paramètre $r$, nous avons estimé le dépôt des sédiments dans la retenue d'eau de la rivière Lobo à l'aide de six modèles. 


\section{Thème 2 - Dynamique sédimentaire}

Ces modélisations avaient pour objectif de démontrer que les dépôts de vases s'effectuent essentiellement par le processus de décantation dans la retenue après avoir été transportée par le débit de crue. Les six (6) modèles établis s'avèrent utiles pour la détermination des épaisseurs de dépôts dans la retenue. Les modèles VC-MT et VC-MC donnent exactement les mêmes résultats. Aussi, en l'absence de données bathymétriques plus anciennes, le travail effectué apporte des éléments de réponse, certes partiels, mais présents.

\section{Références}

BOUGUERRAS S.-A., BOUANANI A. (2019). Analyse saisonnière et interannuelle de la dynamique des flux en suspension dans le bassin versant de l'oued Boukiou (nordouest de l'Algérie). Géomorphologie: relief, processus, environnement, Vol. 25(2), https://doi.org/10.4000/geomorphologie.13189

GROGA N., AKEDRIN T.N., KOMOE K., THIEGBA K., AKAFFOU D.S., OUATTARA A. (2017). Distribution spatio-saisonnière des cyanobactéries le long du cours d'eaux, la Lobo Haut Sassandra (Daloa, Côte d'Ivoire). Tropicultura, Vol. 4(35), pp 288-299.

KOUASSI L.K., SAITA T., KOUAME I.K., SANCHEZ M.A., KOMATSU T., AKA K. (2013). Numerical simulation of the suspended sediment transport in Taabo Lake (Côte d'Ivoire). In: Communication lors de la 7ème édition des Journées scientifiques de l'Institut International d'Ingénierie de l'Eau et de l'Environnement (2iE), 28 p.

MAIGA A.H., DENYIGBA K., ALLORENT J. (2001). Eutrophisation des petites retenues d'eau en Afrique de l'Ouest : Causes et conséquences : Cas de le retenue d'eau sur la Lobo à Daloa en Côte d'Ivoire. Science et Technologie, Vol. 7, pp 16-29. https://doi.org/10.4314/sset.v7i1.18311

MOURIS K., BECKERS F., HAUN S. (2018). Three-dimensional numerical modeling of hydraulics and morphodynamics of the Schwarzenbach reservoir. E3S Web of Conferences, 40: 03005. https://doi.org/10.1051/e3sconf/20184003005

SANCHEZ M. (2006). Settling velocity of the suspended sediment in three high-energy environments. Ocean Engineering, Vol. 33(5-6), pp 665-678. https://doi.org/10.1016/j.oceaneng.2005.05.009

SANCHEZ M. (2018). Effects of convective-diffusive vertical mixing on the conception of rectangular settling basins. Stochastic Environmental Research and Risk Assessment, Vol. 32(5), pp 1457-1463. https://doi.org/10.1007/s00477-017-1462-4

TEETER A.M. (1986). Vertical Transport in Fine-Grained Suspension and NewlyDeposited Sediment. In: Estuarine Cohesive Sediment Dynamics, ed. MEHTA A.J., Springer New York, pp 170-191. https://doi.org/10.1007/978-1-4612-4936-8_9 